\title{
Aleitamento materno: orientações recebidas no pré-natal, parto e puerpério
}

\section{Breastfeeding: guidance received in prenatal care, delivery and postpartum care}

\author{
Mayara Caroline Barbieri' ${ }^{1}$, Luciana Olga Bercini²; Karina Jullyana De Melo \\ Brondani $^{3}$; Rosângela Aparecida Pimenta Ferrari ${ }^{4}$; Mauren Teresa Grubisich \\ Mendes Tacla ${ }^{5}$; Flávia Lopes Sant'anna ${ }^{6}$
}

\begin{abstract}
Resumo
O objetivo do estudo foi analisar as orientações sobre amamentação dadas pelos profissionais de saúde para as mulheres no pré-natal, parto e puerpério. Estudo quantitativo, descritivo, desenvolvido na Regional Pinheiros, Maringá-PR, a partir do cadastro no SisPreNatal, no período de maio a agosto de 2009. As informações foram coletadas por meio de entrevistas em domicílio, utilizando um instrumento estruturado. Participaram da pesquisa 36 mães, sendo que, a maioria recebeu orientações sobre amamentação no pré-natal $(58,3 \%)$, na maternidade $(87,6 \%)$ e nas consultas de enfermagem ao recém-nascido $(84,6 \%)$. A prevalência de amamentação exclusiva foi de $37,5 \%$, mesmo com o término da licença maternidade. Ainda continua baixo o índice de amamentação exclusiva, conforme a recomendação da Organização Mundial da Saúde. Os resultados encontrados poderão contribuir para o monitoramento das ações de saúde e elaboração de novas estratégias na manutenção do aleitamento materno exclusivo.
\end{abstract}

Palavras-chave: Aleitamento materno. Desmame. Educação em saúde. Saúde da mulher.

\begin{abstract}
The aim of the study was to analyze the guidelines considering breastfeeding given by health professionals to women during prenatal care, delivery and postpartum care. Quantitative and descriptive work developed at Regional Pinheiros, Maringá-PR, from the registry in SisPreNatal, from May to August 2009. Data were collected through interviews conducted with parents at home, using a structured instrument. Participants were 36 mothers, most of whom received counseling for breastfeeding during prenatal (58.3\%), maternity $(87.6 \%)$ and in nursing visits to newborn (84.6\%). The prevalence of exclusive breastfeeding was $37.5 \%$, even with the end of maternity leave. The rate is still below the recommended by the World Health Organization for exclusive breastfeeding. The present results may contribute to the monitoring of health actions and development of new strategies in the maintenance of exclusive breastfeeding.
\end{abstract}

Keywords: Breastfeeding. Weaning. Health education. Women's health.

${ }^{1}$ Enfermeira. Residente em Enfermagem em Saúde da Criança, Departamento de Enfermagem, área da Saúde Criança e do Adolescente, Centro de Ciências da Saúde, Universidade Estadual de Londrina (UEL), Londrina, Paraná.

${ }^{2}$ Enfermeira. Doutora Professor da Universidade Estadual de Maringá-UEM. Maringá-PR

${ }^{3}$ Enfermeira. Residente em Enfermagem em Saúde da Criança, Departamento de Enfermagem, área da Saúde Criança e do Adolescente, Centro de Ciências da Saúde, Universidade Estadual de Londrina (UEL), Londrina, Paraná.

${ }^{4}$ Enfermeira. Doutora Professor Adjunto do Departamento de Enfermagem, área da Saúde da Criança e do Adolescente, Centro de Ciências da Saúde, Universidade Estadual de Londrina (UEL), Londrina, Paraná.

${ }^{5}$ Enfermeira. Doutora Professor Adjunto do Departamento de Enfermagem, área da Saúde da Criança e do Adolescente, Centro de Ciências da Saúde, Universidade Estadual de Londrina (UEL), Londrina, Paraná.

${ }^{6}$ Enfermeira. Mestre Professor Assistente do Departamento de Enfermagem em Saúde da Criança e do Adolescente, Centro de Ciências da Saúde, Universidade Estadual de Londrina (UEL), Londrina, Paraná. 


\section{Introdução}

A Organização Mundial da Saúde (OMS) estabeleceu, em 2007, definições em relação ao aleitamento materno (AM) que são reconhecidas no mundo inteiro. Desta forma, o AM é classificado em: Aleitamento Materno Exclusivo (AME), quando a criança recebe somente leite materno(LM) permitindose gotas ou xaropes de vitaminas, suplementos minerais e outros medicamentos; Aleitamento Materno Predominante (AMP), além do LM recebe também água ou bebidas a base de água; Aleitamento Materno Misto ou Parcial (AMM), recebe LM e outros tipos de leite; Aleitamento Materno (AM), independente de receber ou não outros alimentos; e Aleitamento Materno Complementado (AMC), além do LM, qualquer alimento sólido ou semi-sólido com a finalidade de complementá-lo (BRASIL, 2009a).

OAM é a estratégia isolada que mais previne mortes infantis, além de promover a saúde física, mental e psíquica da criança e da mulher que amamenta. Recomenda-se o AME nos primeiros seis meses e AMC por dois anos ou mais (BRASIL, 2009a).

A amamentação tem ação importante para o lactente na proteção contra infecções, diarreia, doenças respiratórias, autoimunes, celíaca e de Crohn, linfomas, diabetes mellitus, entre outras. Também permite seu crescimento e desenvolvimento saudável, fortalecendo o vínculo afetivo entre mãe e filho, além de reduzir o índice de mortalidade infantil e gerar benefícios não só para as crianças, mas também para a nutriz, uma vez que, esta ação produz benefícios econômicos, diminui a ocorrência de alguns tipos de fraturas ósseas e morte por artrite reumatoide, além de câncer de ovários e mamas (MARQUES; COTTA; PRIORE, 2011).

Todavia, a prevalência de AME ainda é baixa, como pode ser observado na pesquisa realizada nas capitais brasileiras e Distrito Federal, em 2008, na qual se detectou que o índice de AME em menores de seis meses foi de 41,0\%. A duração mediana do AME foi de 1,8 meses e a duração mediana do AM de 341,6 dias (VENANCIO et al., 2010).
Assim, novas abordagens devem ser pensadas como uma valorização das ações de promoção, proteção e apoio ao AM como tem acontecido no âmbito hospitalar, por meio da Iniciativa Hospital Amigo da Criança (IHAC), criada em 1992, incorporada pelo Ministério da Saúde como ação prioritária (PEREIRA et al., 2010). Nestes hospitais, a instituição tornase referência em amamentação para seu município, região e estado. A equipe de saúde é mobilizada e colocam em prática os "Dez passos para o sucesso do aleitamento materno" proposto pela OMS e pelo Fundo das Nações Unidas para a Infầncia (BRASIL, 2011).

No foco da saúde materno infantil, o apoio ao AM é uma das principais ações desempenhadas pela atenção básica de saúde. No Brasil, um exemplo deste incentivo na rede pública de saúde para o aumento das taxas de AM foi a criação da Iniciativa Unidade Básica Amiga da Amamentação (IUBAA), devido ao relevante papel da Unidade Básica de Saúde (UBS) na assistência às gestantes, puérperas e bebês, A IUBAA tem como objetivo a promoção, proteção e apoio ao AM, mediante o acolhimento e valorização das preocupações e dúvidas das lactantes e dos seus familiares (GUIMARÃES et al., 2012).

Os profissionais de saúde precisam se apoderar com conhecimentos e habilidades, tanto na prática clínica da lactação como nas habilidades clínicas no aconselhamento (COSTA; ALTAREZ, 2009). As orientações sobre AM não se limitam à assistência no pré-natal, mas se estende para a área hospitalar, pré-parto, parto e puerpério. Nesse sentido, destacase a importância de que a equipe de saúde conheça o cotidiano materno e o contexto sociocultural a que elas pertencem, suas dúvidas, medos e expectativas, bem como, mitos e crenças referentes ao AM, para que possam desmistificar práticas consolidadas pelo "senso comum" que influenciam de forma negativa na lactação (BRANDÃO et al., 2012).

Diante do exposto, o presente estudo teve como objetivo analisar as orientações às mulheres, pelos profissionais de saúde, no pré-natal, parto e puerpério. 
Pois estudos apontam que a qualidade da orientação, desde o período pré-natal, favorece a adesão ao AME, que por sua vez oferece ao bebê não só boas condições físicas, mas também psíquicas, afetivas e econômicas.

\section{Metodologia}

Trata-se de um estudo quantitativo e descritivo, com gestantes da Regional Pinheiros, que é constituída por três UBS, em Maringá-PR, no período de maio a agosto de 2009. Para a coleta de dados utilizou-se o cadastro do SisPreNatal. Em seguida, foi realizada visita domiciliar (VD), previamente agendada, no mês em que a criança completou seis meses de vida. Utilizou-se um instrumento com questões fechadas e abertas referentes ao: perfil sociodemográfico da usuária, escolaridade, profissão, renda, situação conjugal, número de filhos, saúde no período gravídicopuerperal, local do pré-natal, número de consultas e tipo de parto. Também foram consultados os dados da Carteira da Gestante e da Carteira da Criança.

Foram consideradas perdas os seguintes casos: número de telefone inexistente ou por não pertencer mais a mãe, seguido de três tentativas de VD durante a semana e em horários diferentes. Foram excluídas as participantes que sofreram aborto, óbito do bebê e mudança de endereço.

Para a análise dos dados utilizou-se a planilha eletrônica do Excel e, posteriormente, estes foram analisados com o auxílio do Software Statistica 7.1, sendo apresentados em tabelas e figuras. A análise descritiva foi realizada por meio da distribuição percentual das variáveis qualitativas ou categóricas e para as variáveis quantitativas, o cálculo das medidas resumo como medianas, médias e desvios padrão.

O presente estudo recebeu autorização da Secretaria de Saúde de Maringá e parecer favorável do Comitê de Ética em Pesquisa da Universidade Estadual de Maringá, $n^{\circ}$ 256/2011, CAAE: 0176.0.093.00010 , bem como, a permissão das mulheres mediante a assinatura do termo de consentimento livre e esclarecido.

\section{Resultados}

No período do estudo haviam cadastradas no SisPreNatal 107 gestantes, mas seis meses após captouse 48 , sendo que 36 participaram da pesquisa e 12 se recusaram. Os dados referentes à caracterização sociodemográfica, indicados na Tabela 1, mostra maior frequência de mães $(72,2 \%)$ entre a idade de 20 e 34 anos. A média de idade das participantes foi de 23,9 anos, sendo a idade mínima de 15 e a máxima de 39 anos. A maioria (66,7\%) das mães era casada. A escolaridade para aproximadamente $64,0 \%$ era o ensino médio. Quase 67,0\% tinha renda familiar de 1 a 2 salários mínimos. Considerando o trabalho fora de casa no período da gestação, foi verificado que a metade das mulheres $(50,0 \%)$ possuía trabalho formal. Das que trabalhavam fora de casa, todas usufruíram o direito à licença maternidade, sendo que uma $(5,6 \%)$ gozou três meses de licença; 14 (77,7\%) quatro meses; duas $(11,1 \%)$ cinco meses e uma mãe $(5,6 \%)$ seis meses. 
Tabela 1 - Caracterização sociodemográfica das mulheres atendidas pela Regional Pinheiros, Maringá, Paraná, 2009.

\begin{tabular}{|l|c|r|}
\hline & $\mathbf{N}$ & $\mathbf{\%}$ \\
\hline Características maternas sociodemográficas & $\mathbf{3 6}$ & $\mathbf{1 0 0 , 0}$ \\
\hline Idade (anos) & & \\
\hline$\leq 19$ & 26 & 13,9 \\
\hline 20 a 34 & 5 & 72,2 \\
\hline$\geq 35$ & & 13,9 \\
\hline Situação Conjugal & 24 & 66,7 \\
\hline Casada & 7 & 19,4 \\
\hline União consensual & 5 & 13,9 \\
\hline Solteira & & \\
\hline Escolaridade & 10 & 27,8 \\
\hline Ensino fundamental & 23 & 63,9 \\
\hline Ensino médio & 3 & 8,3 \\
\hline Ensino superior & & \\
\hline Renda Familiar (salários mínimos) & 24 & 66,7 \\
\hline$\leq 2$ & 12 & 33,3 \\
\hline$\geq 3$ & & \\
\hline
\end{tabular}

Fonte: Autor.

Na tabela 2 observa-se que a nuliparidade $(52,8 \%)$ foi mais frequente. Quase 90,0\% frequentaram mais de sete consultas durante o pré-natal e cerca de 70,0\% das mulheres realizaram parto cirúrgico. Menos da metade $(47,2 \%)$ havia amamentado outro filho, sendo que, $15(88,2 \%)$ afirmaram que essa experiência anterior foi positiva. Dentre as mães que apresentavam experiência com amamentação a média de AME foi de 126,9 dias e para aquelas que não apresentavam experiência a média foi 108,3 dias. A maioria $(65,0 \%)$ das mães amamentou na primeira hora de vida do recém-nascido.

Tabela 2 - História obstétrica pregressa, do acompanhamento do pré-natal e tipo de parto de mulheres atendidas pela Regional Pinheiros, Maringá, Paraná, 2009.

\begin{tabular}{|l|c|c|}
\hline & $\mathbf{n}$ & $\mathbf{\%}$ \\
\hline Características obstétricas pregressa, pré-natal e parto. & $\mathbf{3 6}$ & $\mathbf{1 0 0 , 0}$ \\
\hline Paridade & & \\
\hline Nulípara & 19 & 52,8 \\
\hline Multípara & 17 & 47,2 \\
\hline Número de consultas no pré-natal & & \\
\hline$\leq 6$ & 1 & 2,8 \\
\hline$\geq 7$ & 35 & 97,2 \\
\hline Tipo de parto & & \\
\hline Cirúrgico & 25 & 69,4 \\
\hline Vaginal & 11 & 30,6 \\
\hline Experiência anterior em amamentar & & \\
\hline Não & 19 & 52,8 \\
\hline Sim & 17 & 47,2 \\
\hline
\end{tabular}

Fonte: Autor. 
Das 36 participantes, $21(58,3 \%)$ receberam orientações sobre amamentação durante o prénatal. Dentre as informações, o tempo de AME, a importância, o posicionamento e pega foram as mais referidas pelas mães. Na maternidade, a grande maioria das mães $(83,3 \%)$ recebeu orientações sobre amamentação, sendo que apenas seis $(16,7 \%)$ não foram orientadas sobre o tema (Tabela 3).

Em relação às orientações na consulta de enfermagem à criança na UBS, $26(65,0 \%)$ foram consultadas e $14(35,0 \%)$ não a fizeram. A maior parte $(84,6 \%)$ das mães que compareceram nas consultas foram orientadas sobre amamentação e quatro $(15,4 \%)$ não.

Tabela 3 - Orientações dos profissionais para as mulheres, sobre amamentação, no prénatal, na maternidade e consulta de enfermagem na UBS, Regional Pinheiros. MaringáParaná, 2009.

\begin{tabular}{|lccccc|}
\hline $\begin{array}{l}\text { Orientações dos profissionais sobre ama- } \\
\text { mentação* }\end{array}$ & $\begin{array}{l}\text { Pré-natal } \\
\text { UBS }\end{array}$ & Maternidade & $\begin{array}{c}\text { Consulta de } \\
\text { enfermagem } \\
\text { na UBS }\end{array}$ \\
\hline & $\mathbf{n}$ & $\mathbf{\%}$ & $\mathbf{n}$ & $\mathbf{\%}$ & $\mathbf{n}$ \\
\hline Importância & 16 & 20,0 & 20 & 21,5 & 18 \\
\hline Tempo de AME & 17 & 21,3 & 19 & 20,4 & 16 \\
\hline Tempo de AMM & 10 & 12,5 & 8 & 8,7 & 11 \\
\hline Problemas mais comuns & 13 & 16,2 & 15 & 16,1 & 13 \\
\hline Posicionamento e pega correta & 16 & 20,0 & 23 & 24,8 & 10 \\
\hline Bicos artificiais & 6 & 7,5 & 7 & 7,5 & 6 \\
\hline Cuidados com as mamas & 2 & 2,5 & 1 & 1,0 & - \\
\hline
\end{tabular}

*Múltiplas respostas

Fonte: Autor.

Vale referir que as orientações no pré-natal foram transmitidas por enfermeiros em 50,0\% dos casos, $38,4 \%$ por médicos, $7,7 \%$ por alunos de enfermagem e 3,9\% por agentes comunitários de saúde (ACS). Na maternidade, a maioria $(87,6 \%)$ das orientações foi feita por enfermeiros, o restante por obstetras $(6,2 \%)$, pediatras $(3,1 \%)$ e técnico ou auxiliar de enfermagem $(3,1 \%)$. Nas consultas de enfermagem na UBS, 21 mulheres (52,5\%) afirmaram ter recebido visita de algum profissional da UBS, sendo cinco $(23,9 \%)$ na primeira semana de vida do bebê, $11(52,3 \%)$ nos primeiros 15 dias, três $(14,2 \%)$ no primeiro mês e duas $(9,6 \%)$ seis meses após o parto. Chama a atenção que 19 (47,5\%) mulheres não foram visitadas por profissionais da UBS no período inicial da amamentação. Também vale referir que $22(61,1 \%)$ das mulheres entrevistadas, buscaram informações sobre amamentação por outros meios, sendo 50,0\% com amigos e parentes, $36,4 \%$ na internet e $13,6 \%$ em livros e revistas.

A figura 1 mostra a prevalência de AME ao longo dos seis primeiros meses de vida a qual é caracterizada por uma linha descendente indicando que há diminuição ao longo dos meses, passando de $82,2 \%$ no primeiro mês de vida para $37,5 \%$ no sexto mês, com ênfase a partir dos 120 dias de vida. 
Figura 1 - Prevalência do Aleitamento Materno Exclusivo nos seis meses de vida das crianças das mulheres, Regional Pinheiros. Maringá, Paraná, 2009.

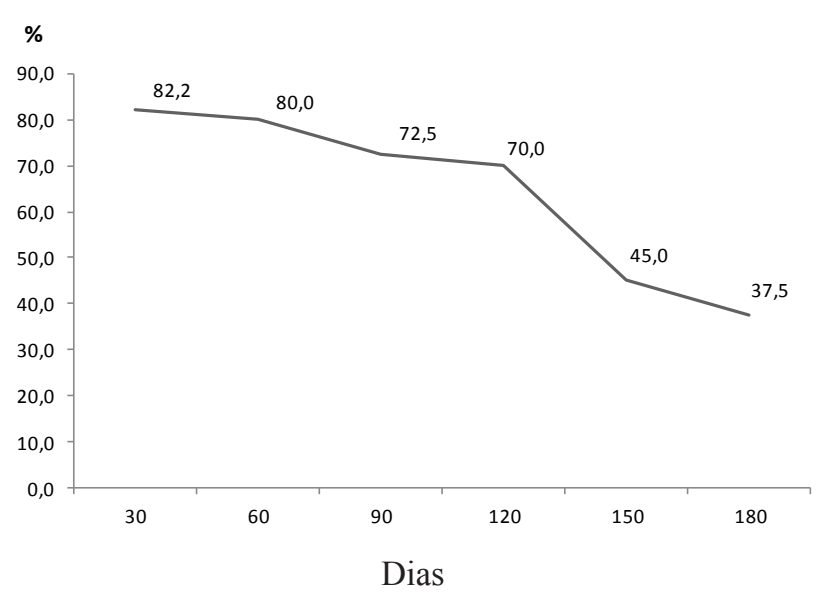

O acréscimo de algum outro tipo de alimento a dieta dos bebês ocorreu em média aos 117 dias, devido ao término da licença maternidade em $32,2 \%$ dos casos, seguido de pouco ganho de peso do bebê $(25,9 \%)$ e por orientação médica $(22,5 \%)$ (Tabela 5).

Tabela 5 - Motivos maternos para interrupção do Aleitamento Materno Exclusivo antes dos seis meses segundo as mulheres atendida na Regional Pinheiros, Maringá, Paraná, 2009.

\begin{tabular}{|l|c|c|}
\hline & $\mathbf{n}$ & $\mathbf{\%}$ \\
\hline Motivos maternos para a interrupção do AME & $\mathbf{3 6}$ & $\mathbf{1 0 0 , 0}$ \\
\hline \hline Término da licença maternidade & 10 & 32,2 \\
\hline Orientação médica & 15 & 48,4 \\
\hline Problemas com a mama & 4 & 13,0 \\
\hline \hline Quis oferecer outros alimentos & 2 & 6,4 \\
\hline
\end{tabular}

Fonte: Autor.

\section{Discussão}

O AME continua sendo um desafio, uma vez que, o índice nas diferentes regiões brasileiras continua aquém do estipulado pela OMS e Ministério da Saúde que é de $100 \%$ até o sexto mês de vida.

Estudos apontam que as condições sociais maternas influenciam no desmame, já que as jovens ou adolescentes são as que menos tempo amamentam, bem como, aquelas que não têm companheiro e/ou apresentam baixa renda. Um dado relevante na presente pesquisa foi a situação conjugal, pois a maior parte que amamentou $(86,1 \%)$ tinha companheiro. Este dado vem de concordância com outro estudo realizado no Rio de Janeiro, onde o número de mulheres em AME era superior entre aquelas cujo pai do bebê morava com a família (PEREIRA et al., 2010).
Onível de escolaridadeé outro fator que tem influência na duração doAME, pois mães com nível de escolaridade mais baixa tendem a introduzir precocemente os alimentos. Nesta pesquisa, as mães com escolaridade até o ensino fundamental, a média de amamentação foi de 61 dias (FALEIROS; TREZZA; CARANDINA 2006). Nas regiões brasileiras mais desenvolvidas, as mulheres mais instruídas, de melhor nível socioeconômico, amamentam por mais tempo. Por outro lado, aquelas com baixa renda realizam menos consultas de pré-natal ou as iniciam tardiamente, amamentando por menos tempo (FALEIROS; TREZZA; CARANDINA 2006).

A média de dias de AME das mães primigestas se apresentou menor se comparado com as mães que já apresentavam experiência anterior. Este dado é confirmado nos resultados de outra pesquisa que 
mostrou que mães com mais idade e maior número de filhos podem ter mais experiência ou maturidade para cuidar e amamentar seus filhos, pois a experiência prévia se encontra associada com uma maior duração da amamentação (BAKOULA et al., 2007). Estes resultados remetem para a importância do incentivo e promoção do AME desde o pré-natal, pelos profissionais de saúde, principalmente para as primigestas. Este apoio deve partir desde o início da gestação, preferencialmente através das Unidades Básicas de Saúde, que devem funcionar como porta de entrada para o sistema de saúde, garantindo resolutividade e atendimento integral, com foco na promoção do aleitamento materno e prevenção de agravos que podem ser evitados com a prática do AME (BATISTELA; GUERREIRO; ROSSETTO, 2008).

Embora o presente estudo mostre que a maioria das mães realizou parto cirúrgico, muitas delas $(65,0 \%)$ amamentaram na primeira hora de vida do recém-nascido. Este resultado foi semelhante ao encontrado em outra pesquisa, realizada nas capitais brasileiras, que foi de 63,0\% (BRASIL, 2009b). Esta prática é recomendada pela OMS por ser uma estratégia de promoção, proteção e apoio ao AM, além de corresponder ao quarto passo da IHAC (LEVY; BÉRTOLO, 2008).

Quanto às orientações realizadas no pré-natal no presente estudo, a maior parte delas foi feita por enfermeiros, resultado semelhante foi verificado em outra pesquisa que identificou que este profissional é o que mais realiza ações de promoção ao aleitamento materno no serviço de saúde (CALDEIRA et al., 2007). Quase a totalidade das mulheres, do presente estudo, referiu ter recebido orientações sobre AM na maternidade. Tem sido relatado que neste momento é de suma importância tal prática, pois a partir do nascimento da criança a mulher iniciará a prática de amamentação, o que favorece intervenções conforme as necessidades de cada uma. A primeira semana de vida é o momento propício para estimular e auxiliar a mulher nas dificuldades com o aleitamento materno exclusivo (SOUZA et al., 2011).

No que se refere à prevalência do $\mathrm{AM}$, entre as mulheres do presente estudo, também foi baixa tal qual de outras pesquisas nas diferentes regiões brasileiras (BRASIL, 2009b).
A principal causa de abandono da amamentação foi o término da licença a maternidade, que foi alterada pela legislação, sendo ampliada para seis meses, de acordo com a regulamentação da Lei 11.770/2008 que ocorreu no final de dezembro de 2009, produzindo efeitos a partir de $1^{\circ}$ de janeiro de 2010 (BRASIL, 2008). Esse decreto passa a entrar em concordância com o que é orientado pelo Ministério da Saúde, ou seja, coincide com o término do período de AME.

Os resultados obtidos na Regional Pinheiros revelaram que apesar da maioria das mulheres terem recebido orientações no pré-natal, uma parcela importante referiu não tê-las recebido e também não participarem de grupos de gestantes, práticas reconhecidamente importantes para o sucesso do AME. Dentre as orientações recebidas no prénatal, destacam-se aquelas relacionadas ao tempo de AME, na maternidade as orientações sobre posicionamento e pega correta foram as mais citadas e na consulta de enfermagem na UBS quanto a importância do AME. As orientações feitas durante o pré-natal e maternidade foram dadas por diversos profissionais, porém houve destaque para o profissional enfermeiro. Além desses profissionais os médicos, alunos de enfermagem, obstetras, ACS, pediatras e técnico ou auxiliar de enfermagem também foram citados como orientadores de informações sobre o AM.

A prevalência de $\mathrm{AME}$ aos seis meses foi aquém do preconizado pela OMS, sendo que a interrupção teve como principais justificativas o término da licença maternidade, o pouco ganho de peso do bebê e a orientação médica.

A amamentação embora seja um ato natural, é também um comportamento, e como tal, pode ser aprendido. Por isso, são necessários profissionais da saúde que encorajem e apoiem as mães para que essas iniciem e mantenham a amamentação exclusiva nos primeiros seis meses da criança e que introduza, no período correto, a alimentação complementar adequada. Os resultados encontrados além de revelarem a situação do AME nas UBS em estudo, poderão contribuir para o monitoramento das ações de saúde e para a elaboração de novas estratégias em relação ao aleitamento materno, visando aumentar as taxas de amamentação. 


\section{Referências}

BAKOULA, C.; VELTSISTA,A.; PREZERAKOU,A.; MOUSTAKI, M.; FRETZAYAS, A.; NICOLAIDOU, P. Working mothers breastfeed babies more than housewives. Acta Paediatrica, Oslo, v. 96, n. 4, p. 510515, 2007. Disponível em: <http://www.ncbi.nlm.nih. gov/pubmed/17391468>. Acesso em: 30 jul. 2013.

BATISTELA, S.; GUERREIRO, N. P.; ROSSETTO, E. G. Os motivos de procura pelo Pronto Socorro Pediátrico de um Hospital Universitário referido pelos pais ou responsáveis. Semina: Ciências Biológicas e da Saúde, Londrina, v. 29, n. 2, p. 121-130. 2008.

BRANDÃO, E. C.; SILVA, G. R. F.; GOUVEIA, M. T. O.; SOARES, L. S.; Caracterização da comunicação no aconselhamento em amamentação. Revista Eletrônica de Enfermagem, v. 14, n. 2, p. 355-365, 2012. Disponível em: <http://www.fen.ufg.br/fen_revista/v14/n2/pdf/ v14n2a16.pdf $>$. Acesso em: 30 jul. 2013.

BRASIL. Ministério da Saúde. Aleitamento materno $e$ alimentação complementar: normas e manuais técnicos. Brasília, 2009a. (Cadernos de Atenção Básica, n. 23).

Iniciativa hospital amigo da criança. Brasília, 2011. Disponível em: <http://bvsms.saude.gov.br/bvs/ publicacoes/iniciativa_hospital_amigo_crianca.pdf $>$. Acesso em: 1 fev. 2012.

Lei $n^{\circ} 11.770$ de 9 de setembro de 2008. Cria o Programa Empresa Cidadã, destinado à prorrogação da licença-maternidade mediante concessão de incentivo fiscal, e altera a Lei $\mathrm{n}^{\mathrm{o}} 8.212$, de 24 de julho de 1991. Disponível em: <http:/www.planalto.gov. br/ccivil_03/_ato2007-2010/2008/lei/111770.htm>. Acesso em: $1 \overline{5}$ jul. 2013.

Secretaria de Atenção à Saúde. II pesquisa de prevalência de aleitamento materno nas capitais brasileiras e Distrito Federal. Brasília, 2009b.

CALDEIRA; P. A.; AGUIAR, G. N.; MAGALHÃES, W.A. C.; FAGUNDES, G. C. Conhecimentos e práticas de promoção do aleitamento materno em Equipes de Saúde da Família em Montes Claros, Minas Gerais, Brasil. Cadernos de Saúde Pública, Rio de Janeiro, v. 23, n. 8, p. 1965-1970, 2007.
COSTA, M. A.; ALTAREZ, C. A. Incentivo ao aleitamento materno nas Unidades Básicas de Saúde do município de Farol, Paraná. SaBios: Revista de Saúde e Biologia, Campo Mourão, v. 4, n. 2, p. 6-13, 2009.

FALEIROS,F.T.V.;TREZZA,E.M.C.;CARANDINA, L. Aleitamento materno: fatores de influência na sua decisão e duração. Revista de Nutrição, Campinas, v. 19, n. 5, p. 623-630. 2006.

GUIMARÃES, L.A. O. P.; MARÇAL, F.; ZUFFI, F. B.; RIBEIRO, M. C.; RODRIGUES, L. R.; MACHADO, M. O. F. Pet-Saúde na identificação do conhecimento de gestantes sobre aleitamento materno. Ciência Cuidado e Saúde, Maringá, v. 11, n. 3, p. 454-462, 2012.

LEVY; L.; BÉRTOLO, H. Manual de aleitamento materno. 2008. Disponível em: <www.unicef.pt/docs/ manual_aleitamento.pdf $>$. Acesso em: 6 ago. 2013.

MARQUES, E.; S.; COTTA, R.; M.; M.; PRIORE, S. E. Mitos e crenças sobre o aleitamento materno. Ciência e. Saúde Coletiva, Rio de Janeiro, v. 16, n. 5, p. 2461-2468, 2011. Disponível em: <http://www. scielo.br/scielo.php?script $=$ sci arttext\&pid $=$ S1413$81232011000500015 \& I n g=$ en\&nrm=iso $>$. Acesso em: 6 ago 2013.

PEREIRA, R. S. V.; OLIVEIRA, M. I. C.; ANDRADE. C. L. T.; BRITO, A. S. Fatores associados ao aleitamento materno exclusivo: o papel do cuidado na atenção básica. Cadernos de Saúde Pública, Rio de Janeiro, v. 26, n. 12, p. 2343-2354. 2010.

SOUZA, M. H. N.; GOMESLL, T. N. C.; PAZLL, E. P. A.; TRINDADE, C. S.; VERAS, R. C. C. Estratégia acolhimento mãe-bebê: aspectos relacionados à clientela atendida em uma unidade básica de saúde do município do Rio de Janeiro. Escola Anna Nery, Rio de Janeiro, v. 15, n. 4, p. 671-677, 2011.

VENANCIO, S. I.; ESCUDER, M. M. L.; SALDIVA, S. R. D. M.; GIUGLIANE, E. R. J.A prática do aleitamento materno nas capitais brasileiras e Distrito Federal: situação atual e avanços. Jornal de Pediatria, Rio de Janeiro, v. 86, n. 4, 2010. Disponível em: <http://www. scielo.br/scielo.php?script=sci_arttext\&pid=S0021$75572010000400012 \& I n g=$ en\&nrm $=$ iso $>$. Acesso em: 6 ago 2013.

Recebido em: 24 set. 2013. Aceito em: 22 mai. 2014. 